\title{
Ethics of Glaucoma Widgets
}

\author{
Tanuj Dada ${ }^{1}$, Priyanka Ramesh ${ }^{2}$, Anin Sethi $^{3}$, Shibal Bhartiya ${ }^{4}$ \\ Journal of Current Glaucoma Practice (2020): 10.5005/jp-journals-10078-1288
}

Ethics is knowing the difference between what you have a right to do and what is right to do.

-Potter Stewart

Which surgical procedure to adopt for a glaucoma patient? In the last decade, the options available for managing a glaucoma patient have exploded and currently, there is no standardized way of treatment available with wide variations based on surgeon training, patient affordability, and regional availability of surgical devices. Even though a high intraocular pressure (IOP) is not the defining prerequisite for glaucoma diagnosis, its lowering is the only definitive treatment to prevent the progression of glaucomatous optic neuropathy. ${ }^{1}$ Additionally, surgery which had hitherto been reserved for patients with advanced glaucoma and those with uncontrolled IOP on maximal tolerable medical therapy has shifted toward early glaucoma and even ocular hypertension with a focus on doing away with medical therapy and improving the quality of life (QoL) measures. ${ }^{2}$ Trabeculectomy has a long and proven track record as the gold standard, with glaucoma drainage devices (GDD)/tubes reserved for eyes with refractory glaucoma or failed trabeculectomy, although there is a trend for more tube surgeries after the TVT study. ${ }^{3}$ Even though trabeculectomy and tube have the best IOP lowering capability, they do have a significant risk of sight-threatening complications.

In the last decade, a new generation of glaucoma implants has gained momentum, bridging the glaucoma management spectrum: the safety of topical therapy and the efficacy of conventional glaucoma surgeries. These implants are broadly categorized as MIGS- "Minimally Invasive Glaucoma Surgery". The MIGS implants are ideally inserted $a b$ interno without any conjunctival incision. However, ab-externo devices with minimal to no scleral dissection have also been included in the broad classification of MIGS. ${ }^{4}$ These devices are proposed to have a better safety profile than conventional filtering surgery/tubes, with a moderate IOP lowering efficacy and a shorter surgical time. ${ }^{5}$

For the widespread utilization and acceptance of any new surgical modality as a replacement of the existing standard of care, the former should display a similar efficacy, a better safety profile, and cost-effectiveness for the global population. With the introduction of many new implants, the ophthalmic community needs to pay adequate attention to the ethical implications of their use on patients.

The central issue with trabeculectomy surgery has been the long-term complications (bleb leak, hypotony, blebitis, endophthalmitis) associated with the use of mitomycin $C$ as an antifibrotic agent. However, the incidence of these complications can be lowered by simply reducing the dose of mitomycin $C$ to $0.1 / 0.2$ mg for 3 minutes; instead of using $0.4 \mathrm{mg}$ for 3 to 5 minutes, as reported in some major surgical trials. ${ }^{6} \mathrm{~A}$ bioengineered collagen implant called "Ologen", was proposed as an alternative to mitomycin C. It was engineered to modulate wound healing and to
${ }^{1-3}$ Department of Ophthalmology, Dr RP Centre for Ophthalmic Sciences, All India Institute of Medical Sciences, New Delhi, India

${ }^{4}$ Glaucoma Services, Department of Ophthalmology, Fortis Memorial Research Institute, Gurugram, Haryana, India

Corresponding Author: Tanuj Dada, Department of Ophthalmology, Dr RP Centre for Ophthalmic Sciences, All India Institute of Medical Sciences, New Delhi, India, Phone: +91 11-26593101, e-mail: tanujdada@gmail.com

How to cite this article: Dada T, Ramesh P, Sethi A, et al. Ethics of Glaucoma Widgets. J Curr Glaucoma Pract 2020;14(3):77-80.

Source of support: Nil

Conflict of interest: None

provide a scaffold for the growth of the fibroblasts; thus, preventing closure of the ostium and the flap. However, trabeculectomy with Ologen was found to have poorer outcomes, when compared with trabeculectomy with mitomycin C; and additionally, it was reported that there was no benefit of adding Ologen to an MMC augmented trabeculectomy. ${ }^{7-10}$

The advent of GDD has brought new hope for the management of refractory glaucoma and failed trabeculectomy. ${ }^{11}$ But the use of these implants in surgery naïve eyes was found to have lessdesirable outcomes as compared to those of MMC augmented trabeculectomy. In the PTVT study, trabeculectomy was found to have a higher success rate, less need for medications, and lower mean IOP 3 years after surgery. ${ }^{6}$ Any filtering surgery, tube or trabeculectomy, is bound to fail sooner or later, hence, the possibility of a re-surgery cannot be overlooked. In our opinion, the primary surgical procedure should allow the conservation of healthy conjunctiva for future filtering surgery(s). Additionally, tubes must never be advocated as primary surgery for phakic patients due to the high risk of progressive corneal endothelial cell loss. ${ }^{12}$ If we consider the Medicare data, the risk of severe sight-threatening complications was three times more with GDD as compared to trabeculectomy. The 6-year cumulative incidence of both serious and less serious adverse effects was more with GDD. ${ }^{13}$ Other than having a higher rate of complications, the GDDs were less cost-effective as compared to trabeculectomy, making them an inferior option as the primary surgical procedure. ${ }^{14}$ However, due to insurance concerns related to reimbursement for postoperative visits, some ophthalmic centers have started advocating tube implantation as the primary surgery, as it may require less frequent visits in the initial postoperative period. We feel that offering tube implantation as the first line of surgical intervention to patients is unethical and should be reserved for select cases, i.e., refractory glaucoma, high risk of trabeculectomy failure, or eyes with a failed trabeculectomy. The patient should preferably be pseudophakic and the ciliary sulcus should be chosen as the site of implantation to decrease the risk of corneal endothelial damage. ${ }^{15,16}$ 
Minimally invasive glaucoma surgery has been widely promoted, as a less invasive alternative for mild to moderate glaucoma and also to reduce dependency on glaucoma medications. ${ }^{5}$ They are devices that work either by enhancing trabecular outflow, by deroofing the Schlemm's canal (GATT, KDB) or bypassing the abnormal trabecular meshwork by inserting implants such as iStent and Hydrus; by shunting the aqueous flow from the anterior chamber into the subconjunctival space with the Xen/ Preserflo implant or into the suprachoroidal space by implants like Cypass and iStent supra. The iStent (Glaukos Corporation, Laguna Hills, CA, USA) which is injected during cataract surgery was FDA approved in June 2012. Most studies that showed IOP lowering effect were done with a concurrent cataract surgery which, on its own has the potential to lower the IOP. ${ }^{17}$ In a study done in mild to moderate open-angle glaucoma, it was found that iStent reduced the IOP to $\leq 21 \mathrm{~mm} \mathrm{Hg}$ in $72 \%$ whereas, cataract surgery alone showed a reduction in $50 \%$ of the cases. ${ }^{18}$ When compared with medical therapy, it was found that even after injecting two iStents, the efficacy was comparable to that of medical therapy alone. ${ }^{19}$ The iStent also had complications such as hyphema, malposition, and obstruction of the implant. The implant may offer only a 3-10\% additional reduction in IOP as compared to cataract surgery and is indicated only in cases with mild to moderate glaucoma, which can be controlled by one or two medications. ${ }^{17}$ Having said this, the location of the implantation of the iStent also plays an important role in its functioning. The aqueous humor outflow into the aqueous veins is not uniform throughout the circumference of the angle. Studies have shown that the outflow is more prominent nasally as compared to other quadrants, hinting that the iStent has to be placed where there is more drainage and random placement may be futile. ${ }^{20}$ An angiography-guided iStent placement in the future may offer better results.

The Xen implant (Allergan plc, Dublin, Ireland) creates a shunt between the anterior chamber and the subconjunctival space. When compared with trabeculectomy, the Xen implant had no difference in risk of failure as well as safety profile. The needling rate was higher in Xen implant (43.2 vs 30.8\%) as compared to trabeculectomy and the reoperation rate was also higher in the Xen group. ${ }^{21}$ This implant is not without its own set of complications such as blockage of the tip, migration of the stent, hyphema, subconjunctival bleeding, breakage during manipulation especially during needling, and displacement of the stent on the strenuous rubbing of eyes. ${ }^{22,23}$ The implantation of the implant itself can have complications such as malposition, which will require additional manipulations leading to trauma and hyphema and eventually, blockage of the ostium warranting a surgical displacement of the clot. ${ }^{24}$ Some batches of the implant have been voluntarily recalled by Allergan over issues due to the presence of trace amounts of residual polishing compounds. ${ }^{25}$ Microfluidic rabbit eyes study has found that the resistance to aqueous outflow was more with $a b$ interno subconjunctival drainage devices. The bleb formed was more variable and smaller with this approach, and in addition, this technique resulted in spikes of IOP $>21 \mathrm{~mm} \mathrm{Hg} .{ }^{26}$ With no definitive benefit over trabeculectomy and with the absence of long-term data especially on the safety of the implant, one wonders if its use is justified, especially with a localized injection of mitomycin C, which can have serious sight-threatening complications in the long-term.

An implant that was recalled from the market was the CyPass (Alcon Inc., Fort Worth, TX, USA). Alcon recalled CyPass after reports of endothelial cell loss which continued for at least 5 years after the implantation of the device. ${ }^{27,28}$ When patients come to know that the implant inside their eye is potentially hazardous and has been recalled, it can lead to unwarranted anxiety and psychological problems. The ExPress glaucoma filtration device is another implant (advocated as an alternative to trabeculectomy), that is injected $a b$-externo allowing drainage of the aqueous from the anterior chamber into the subconjunctival space. The initial unguarded implantation had many complications such as hypotony, extrusion, endophthalmitis, and erosion causing the company to recommend its implantation under a partial thickness scleral flap. ${ }^{29}$ When compared to trabeculectomy, at 3 years, IOP between 5 and $18 \mathrm{~mm} \mathrm{Hg}$ was seen in 35\% in the ExPress group and 38\% in the trabeculectomy group. And qualified success was seen in $52 \%$ in the ExPress group as compared to $61 \%$ in the trabeculectomy group. ${ }^{30}$ A randomized control trial done for the postoperative complications post-ExPress implantation showed that the endothelial cell loss was much higher than trabeculectomy at 6 months, 1 year, and 2 years. There were no differences in the early complications such as choroidal detachment, flat anterior chamber, hypotony as well as no difference in the IOP and needling rate. It was also found that the implant had a risk of iris touch, which was more likely in eyes with a shallow anterior chamber preoperatively. ${ }^{31,32}$ There was also a report of chronic pain due to an iris embedded implant, that needed to be removed; as well as reports of blockage, extrusion, and dislocation of the implant. ${ }^{33,34}$ Although, advocated as a substitute for trabeculectomy, the ExPress is no longer being used, primarily because of higher cost, a higher rate of failure and complications. ${ }^{35}$

Trabectome is a device used for ab interno trabeculotomy which uses plasma mediated ablation to expose the Schlemm's canal. It removes the trabecular tissue which gives the maximum resistance to outflow. Though theoretically, it sounds like a good idea, the Mayo clinic series showed less favorable results: at 24 months, the success with the criteria IOP of $<21 \mathrm{~mm} \mathrm{Hg}$ was $62 \%$ but when IOP criteria $<18 \mathrm{~mm} \mathrm{Hg}$ was taken, it fell to just $22 \%$, showing that it is not a good alternative when the target IOP is below the commonly recommended $18 \mathrm{~mm} \mathrm{Hg}$ for any glaucoma patient. The surgery also had a high incidence of hyphema (73\%). ${ }^{36}$ A study was done by Jea et al. also showed lower success rates with ab interno trabeculotomy when compared to trabeculectomy at 2 years ( 22 vs $76 \%$ ) with more number of additional glaucoma surgeries needed in the $a b$ interno trabeculotomy group (43.5 vs $10.8 \%$ ). ${ }^{37}$

A meta-review by Gillmann and Mansouri reported that of the studies considered, GATT reported a decrease in IOP that ranged from $44 \%$ at 12 months, to 37.3 to $55 \%$ at the end of two years. They also reported an incidence of hyphema that ranged from 28 to $38 \%$ in these patients, with more than $15 \%$ experiencing IOP spikes. ${ }^{38}$

The solitary study of PreserFlo (earlier called Innfocus/Arrow) included in the review reported a 50\% IOP reduction at 24 months, with an $80 \%$ decrease in antiglaucoma medication. The authors reported hypotony with shallow anterior chambers in $13 \%$ of patients, $8.7 \%$ of whom also reported a choroidal detachment. ${ }^{39}$

The concerns regarding the IOP lowering efficacy of MIGS has been raised repeatedly. Minimally invasive glaucoma surgery has been labeled as "MEGS" - Minimally effective glaucoma surgery and even as MIGS-Maximal Glaucoma Income Syndicate!!!. As equity, equality and universal access become the basic tenets of ethical healthcare, the economics of any glaucoma surgical intervention must be an integral part of its assessment. As we envision a 10-10-10 goal of any glaucoma surgery, we require a technique that can be done in 10 minutes, provide IOP as low as $10 \mathrm{~mm} \mathrm{Hg}$ and last for 10 
years but we need to add another 10 to this-a cost of 10 dollars, to have a meaningful impact on the global population. The current MIGS techniques miserably fail to reach this goal. ${ }^{40,41}$

Some clinicians argue that MIGS may contribute to a better QoL for glaucoma patients. However, a study done by Pahlitzsch et al. showed that there was no significant difference in the QoL measured by the NEI-VFQ-25 questionnaire between trabeculectomy, iStent, and trabectome. They also found that the trabeculectomy group required a lower number of medications postoperatively which could also influence the QoL. ${ }^{42}$

Other than the efficacy issues with these devices, other key points question the ethics of choosing a particular MIGS implant for a patient. Currently, there are no standardized objective criteria for the implantation of any specific device. There is no clear backing by evidence-based medicine. Most devices are chosen based on the surgeon's own preference, training experience, and comfort. With the lack of clear evidence of benefit to patients, we are forced to question as to whom exactly do these devices benefit?!

With the non-availability of enough high-quality data and many trials being industry-sponsored, along with limited evidence on the economics of these devices, one wonders if the widespread use of these newer implants in patients is ethically correct. In developing countries with a large proportion of the population falling in the low-income group, it has been found that they spend around $60 \%$ of their monthly income on glaucoma therapy. ${ }^{43}$ With the lack of any concrete data on the economic benefits, and the cost of these implants being higher than trabeculectomy, its use in developing countries is controversial. Laupacis et al. published tentative guidelines for the use of any new technology in medicine as five grades of recommendation. The new technology which is both more effective and economical compared to the existing technology is an ideal situation with grade A. Whereas, grade $\mathrm{E}$ is one where the technology is far more expensive as well as less effective than the existing treatment and such technology needs to be rejected. With the available data, it seems the new glaucoma devices fall under this grade, making their use questionable. ${ }^{44}$

We must learn to distinguish between errors of knowledge and breaches of morality. The clinical trial system that we rely so heavily on, is not completely transparent; with irregularities in the trials, especially those which are conducted by the industries. ${ }^{45}$ Concerns regarding drug and medical technology corporations funding and promoting research, and its implications in clinical practice, has been raised. There are studies, where the funding for the consultation fees and travel support have been given by the companies. ${ }^{46}$ Outcomes that are statistically significant with no clinical relevance, are a problem. ${ }^{47}$ A systematic review also pointed out that several studies were funded and designed by the corporations manufacturing these MIGS devices, creating a bias; and it would be dangerous for clinicians to take decisions based on these studies. ${ }^{48}$ It was found that MIGS trials had overall low compliance with the World Glaucoma Association reporting guidelines; only $21.2 \%$ adhered to the definition of success, $37.3 \%$ to the statistical reporting, and $0 \%$ when it came to economic evaluation. Sixty-four percent of the studies had an author who was associated with the company manufacturing the devices, and $24 \%$ had an author who was an employee in the industry. ${ }^{49}$

Especially with the absence of long-term data pertaining to its safety, and new reports of adverse effects prompting the withdrawal of implants, the ethical implications of implanting these in patients, especially those with mild to moderate glaucoma, should be reconsidered. ${ }^{14,25,27,48,50,51}$ There is an immediate need for high quality, long-term studies on these implants with, standardized methodology and reporting, before their widespread use in the population.

\section{References}

1. Weinreb RN, Aung T, Medeiros FA. The pathophysiology and treatment of glaucoma: a review. JAMA 2014;14(311):1901-1911. DOI: 10.1001/jama.2014.3192.

2. Razeghinejad MR, Fudemberg SJ, Spaeth GL. The changing conceptual basis of trabeculectomy: a review of past and current surgical techniques. Surv Ophthalmol 2012;2(57):1-25. DOI: 10.1016/j. survophthal.2011.07.005.

3. Molteno AC. New implant for drainage in glaucoma. Clinical trial $\mathrm{Br}$ J Ophthalmol 1969;53(9):606-615. DOI: 10.1136/bjo.53.9.606.

4. Caprioli J, Kim JH, Friedman DS, et al. Special commentary: supporting innovation for safe and effective minimally invasive glaucoma surgery: summary of a joint meeting of the American Glaucoma Society and the Food and Drug Administration, Washington, DC, February 26, 2014. Ophthalmology 2015;122(9):1795-1801. DOI: 10.1016/j.ophtha.2015.02.029.

5. Pillunat $L E$, Erb $C$, Jünemann $A G$, et al. Micro-invasive glaucoma surgery (MIGS): a review of surgical procedures using stents. Clin Ophthalmol AuckI NZ 2017;11:1583. DOI: 10.2147/OPTH.S135316.

6. Gedde SJ, Feuer WJ, Lim KS, et al. Treatment outcomes in the primary tube versus trabeculectomy study after 3 years of follow-up. Ophthalmology 2020;127(3):333-345. DOI: 10.1016/j. ophtha.2019.10.002.

7. Rosentreter A, Schild AM, Jordan JF, et al. A prospective randomised trial of trabeculectomy using mitomycin $C$ vs an ologen implant in open angle glaucoma. Eye Lond Engl 2010;24(9):1449-1457. DOI: 10.1038/eye.2010.106.

8. Rosentreter A, Gaki S, Cursiefen C, et al. Trabeculectomy using mitomycin $C$ versus an atelocollagen implant: clinical results of a randomized trial and histopathologic findings. Ophthalmol J Int Ophtalmol Int J Ophthalmol Z Augenheilkd 2014;231(3):133-140. DOI: 10.1159/000356400.

9. Boey P-Y, Narayanaswamy A, Zheng C, et al. Imaging of blebs after phacotrabeculectomy with ologen collagen matrix implants. Br J Ophthalmol 2011;95(3):340-344. DOI: 10.1136/bjo.2009.177758.

10. Sen M, Midha N, Sidhu T, et al. Prospective randomized trial comparing mitomycin $\mathrm{C}$ combined with ologen implant versus mitomycin $\mathrm{C}$ alone as adjuvants in trabeculectomy. Ophthalmol Glaucoma 2018;1(2):88-98. DOI: 10.1016/j.ogla.2018.07.003.

11. Gedde SJ, Schiffman JC, Feuer WJ, et al. Treatment outcomes in the tube versus trabeculectomy (TVT) study after five years of follow-up. Am J Ophthalmol 2012;153(5):789-803.e2. DOI: 10.1016/j. ajo.2011.10.026.

12. Janson BJ, Alward WL, Kwon YH, et al. Glaucoma-associated corneal endothelial cell damage: a review. Surv Ophthalmol 2018;63(4):500506. DOI: 10.1016/j.survophthal.2017.11.002.

13. Stein JD, Ruiz D, Belsky D, et al. Longitudinal rates of postoperative adverse outcomes after glaucoma surgery among medicare beneficiaries 1994 to 2005. Ophthalmology 2008;115:1109-1116.e7.

14. Kaplan RI, DeMoraes CG, CioffiGA, etal.Comparative cost-effectiveness of the baerveldt implant, trabeculectomy with mitomycin, and medical treatment. JAMA Ophthalmol 2015;133(5):560-567. DOI: 10.1001/jamaophthalmol.2015.44.

15. Weiner A, Cohn AD, Balasubramaniam M, et al. Glaucoma tube shunt implantation through the ciliary sulcus in pseudophakic eyes with high risk of corneal decompensation. J Glaucoma 2010;19(6):405-411. DOI: 10.1097/IJG.0b013e3181bdb52d.

16. Eslami Y, Mohammadi M, Fakhraie G, et al. Glaucoma valve implantation with tube insertion through the ciliary sulcus in pseudophakic/aphakic eyes. J Glaucoma 2014;23(2):115-118. DOI: 10.1097/IJG.0b013e318265bc0b. 
17. Wellik SR, Dale EA. A review of the iStent ${ }^{\oplus}$ trabecular micro-bypass stent: safety and efficacy. Clin Ophthalmol AuckI NZ 2015;15(9):677684. DOI: $10.2147 /$ OPTH.S57217.

18. Samuelson TW, Katz LJ, Wells JM, et al. Randomized evaluation of the trabecular micro-bypass stent with phacoemulsification in patients with glaucoma and cataract. Ophthalmology 2011;118(3):459-467. DOI: $10.1016 /$ j.ophtha.2010.07.007.

19. Fea AM, Belda Jl, Rękas $M$, et al. Prospective unmasked randomized evaluation of the iStent inject $\left.{ }^{(}\right)$versus two ocular hypotensive agents in patients with primary open-angle glaucoma. Clin Ophthalmol 2014;8:875-882. DOI: 10.2147/OPTH.S59932.

20. Huang AS, Penteado RC, Saha SK, et al. Fluorescein aqueous angiography in live normal human eyes. J Glaucoma 2018;27(11):957964. DOI: 10.1097/IJG.0000000000001042.

21. Schlenker MB, Gulamhusein $\mathrm{H}$, Conrad-Hengerer I, et al. Efficacy, safety, and risk factors for failure of standalone ab interno gelatin microstent implantation versus standalone trabeculectomy. Ophthalmology 2017;124(11):1579-1588. DOI: 10.1016/j.ophtha.2017.05.004.

22. Gupta C, Mathews D. XEN ${ }^{\otimes}$ stent complications: a case series. BMC Ophthalmol 2019;19(1):253. DOI: 10.1186/s12886-019-1267-y.

23. De Gregorio A, Pedrotti E, Russo L, et al. Minimally invasive combined glaucoma and cataract surgery: clinical results of the smallest $a b$ interno gel stent. Int Ophthalmol 2018;38(3):1129-1134. DOI: 10.1007/ s10792-017-0571-x.

24. Pinto Ferreira N, Abegão Pinto L, Marques-Neves C. XEN gel stent internal ostium occlusion: Ab-interno revision. J Glaucoma 2017;26(4):e150-e152. DOI: 10.1097/IJG.0000000000000625.

25. RECALL: XEN 45 Implants by Allergan [Internet]. American Academy of Ophthalmology. 2019 [accessed 2021Jan 26]. Available from: https:// www.aao.org/eye-on-advocacy-article/recall-xen-45-implants-byallergan.

26. Lee RMH, Bouremel Y, Eames I, et al. The implications of an ab interno versus ab externo surgical approach on outflow resistance of a subconjunctival drainage device for intraocular pressure control. Transl Vis Sci Technol 2019;8(3):58. DOI: 10.1167/tvst.8.3.58.

27. UPDATE: Potential Eye Damage from Alcon CyPass Micro-Stent Used to Treat Open-Angle Glaucoma: FDA Safety Communication. FDA [Internet]. 2019 Dec 20 [accessed 2021 Jan 26]; Available from: https://www.fda.gov/medical-devices/safety-communications/ update-potential-eye-damage-alcon-cypass-micro-stent-usedtreat-open-angle-glaucoma-fda-safety.

28. Lass JH, Benetz BA, He J, et al. Corneal endothelial cell loss and morphometric changes 5 years after phacoemulsification with or without CyPass micro-stent. Am J Ophthalmol 2019;208:211-218. DOI: 10.1016/j.ajo.2019.07.016.

29. Sarkisian SR. The ex-Press mini glaucoma shunt: technique and experience. Middle East Afr J Ophthalmol 2009;16(3):134-137. DOI: 10.4103/0974-9233.56226.

30. Gonzalez-Rodriguez JM, Trope GE, Drori-Wagschal L, et al. Comparison of trabeculectomy versus ex-PRESS: 3 -year follow-up. Br J Ophthalmol 2016;100(9):1269-1273. DOI: 10.1136/bjophthalmol-2015-307161.

31. Arimura $S$, Miyake $S$, Iwasaki $K$, et al. Randomised clinical trial for postoperative complications after ex-PRESS implantation versus trabeculectomy with 2-year follow-up. Sci Rep 2018;8(1):16168. DOI: 10.1038/s41598-018-34627-w.

32. Arimura S, Takihara $Y$, Miyake $S$, et al. Randomized clinical trial for early postoperative complications of ex-PRESS implantation versus trabeculectomy: complications postoperatively of ex-PRESS versus trabeculectomy study (CPETS). Sci Rep 2016;6(1):1-7. DOI: 10.1038/ srep26080.

33. Grover DS, Fellman MA, Fellman RL. New ab interno technique for removal of iris-embedded EX-PRESS shunt and chronic eye pain caused by shunt malpositioning. JAMA Ophthalmol 2013;131(10):1356-1358. DOI: 10.1001/jamaophthalmol.2013.4274.

34. Teng CC, Radcliffe N, Huang JE, et al. Ex-PRESS glaucoma shunt dislocation into the anterior chamber. J Glaucoma 2008;17(8):687689. DOI: $10.1097 /$ IJG.0b013e3181666595.

35. Angmo D, Sharma R, Temkar $S$, et al. Evaluation of ExPress glaucoma filtration device in Indian patients with advanced glaucoma. Indian J Ophthalmol 2015;63(5):459. DOI: 10.4103/0301-4738.159894.

36. Ahuja Y, Ma Khin Pyi S, Malihi M, et al. Clinical results of ab interno trabeculotomy using the trabectome for open-angle glaucoma: the mayo clinic series in Rochester, Minnesota. Am J Ophthalmol 2013;156(5):927-935.e2. DOI: 10.1016/j.ajo.2013.06.001.

37. Jea SY, Francis BA, Vakili G, et al. Ab interno trabeculectomy versus trabeculectomy for open-angle glaucoma. Ophthalmology 2012;119(1):36-42. DOI: 10.1016/j.ophtha.2011.06.046.

38. Gillmann K, Mansouri K. Minimally invasive glaucoma surgery: where is the evidence? Asia pac. J Ophthalmol (Phila) 2020;9(3):203-214. DOI: 10.1097/APO.0000000000000294.

39. Pinchuk L, Riss I, Batlle JF, et al. The use of poly(styreneblockisobutylene-block-styrene) as a microshunt to treat glaucoma. Regen Biomater 2016;3(2):137-142. DOI: 10.1093/rb/rbw005.

40. Lee RMH, Bouremel Y, Eames I, et al. Translating minimally invasive glaucoma surgery devices. Clin Transl Sci 2020;13(1):14-25. DOI: 10.1111/cts.12660.

41. Sheheitli H, Tirpack AR, Parrish 2nd RK. Which patients would most likely to benefit: MIGS or MEGS, which one is it? Asia Pac J Ophthalmol (Phila) 2019;8(6):436-440. DOI: 10.1097/APO.0000000000000260.

42. Pahlitzsch M, Klamann MK, Pahlitzsch ML, et al. Is there a change in the quality of life comparing the micro-invasive glaucoma surgery (MIGS) and the filtration technique trabeculectomy in glaucoma patients? Graefes Arch Clin Exp Ophthalmol 2017;255(2):351-357. DOI: 10.1007/s00417-016-3550-4.

43. Lazcano-Gomez G, Ramos-Cadena MLA, Torres-Tamayo M, et al. Cost of glaucoma treatment in a developing country over a 5-year period. Medicine (Baltimore) 2016;95(47):e5341. DOI: 10.1097/ MD.0000000000005341.

44. Laupacis A, Feeny D, Detsky AS, et al. How attractive does a new technology have to be to warrant adoption and utilization? tentative guidelines for using clinical and economic evaluations. Can Med Assoc J 1992;146:473-481.

45. Cohen D. FDA official: "clinical trial system is broken". BMJ 2013;347(dec05 3):f6980. DOI: 10.1136/bmj.f6980.

46. Tanna AP, Rademaker AW, de Moraes CG, et al. Collagen matrix vs mitomycin-C in trabeculectomy and combined phacoemulsification and trabeculectomy: a randomized controlled trial. BMC Ophthalmol 2016;16(1):217. DOI: 10.1186/s12886-016-0393-z.

47. Spence D. Evidence based medicine is broken. BMJ 2014;348(jan03 1):g22. DOI: 10.1136/bmj.g22.

48. Rosdahl JA, Gupta D. Prospective studies of minimally invasive glaucoma surgeries: systematic review and quality assessment. Clin Ophthalmol 2020;14:231-243. DOI: 10.2147/OPTH.S239772.

49. Mathew DJ, McKay BR, Basilious A, et al. Adherence to world glaucoma association guidelines for surgical trials in the era of microinvasive glaucoma surgeries. Ophthalmol Glaucoma 2019;2(2):78-85. DOI: 10.1016/j.ogla.2019.01.007.

50. Agrawal P, Bradshaw SE. Systematic literature review of clinical and economic outcomes of micro-invasive glaucoma surgery (MIGS) in primary open-angle glaucoma. Ophthalmol Ther 2018;7(1):49-73. DOI: 10.1007/s40123-018-0131-0.

51. Nichani P, Popovic MM, Schlenker MB, et al. Micro-invasive glaucoma surgery: a review of 3476 eyes. Surv Ophthalmol 2020. DOI: 10.1016/j. survophthal.2020.09.005S0039-6257(20)30135-1. 